\title{
Obstruction Gastric, CTCAE
}

National Cancer Institute

\section{Source}

National Cancer Institute. Obstruction Gastric, CT CAE. NCI Thesaurus. Code C143710.

A disorder characterized by blockage of the normal flow of the contents in the stomach. 